\section{Percepción del aprendizaje basado en problemas y el desarrollo de capacidades en estudiantes de una Facultad de Estomatología}

\section{Perception of problem-based learning and development of abilities in students of a Faculty of Stomatology}

\section{Resumen}

Objetivo: Determinar la relación existente entre la percepción de estudiantes sobre el uso del aprendizaje basado en problemas y el desarrollo de capacidades en la Facultad de Estomatología de la Universidad Inca Garcilaso de la Vega (UIGV). Materiales y método: Estudio descriptivo, transversal y correlacional. La población fueron estudiantes del quinto ciclo la Facultad de Estomatología de la UIGV del 2015-I, de Prótesis Parcial Removible que cumplieron con los criterios de inclusión (matriculados en la asignatura de Prótesis Parcial Removible del año 2015-I, accesibilidad, disponibilidad horaria y aceptar participar en el trabajo). Se trabajó con 49 alumnos. El instrumento utilizado: Un cuestionario de evaluación. El tenor de las preguntas indagó sobre la percepción que desarrollan los estudiantes sobre la estrategia de aprendizaje basada en problemas y el desarrollo de capacidades que considera haber desarrollado. Resultados: No se encontró correlación entre la dimensión condiciones sobre el uso del modelo didáctico de aprendizaje basado en problemas con el desarrollo de capacidades conceptuales, procedimentales y actitudinales por medio de la prueba de correlación de Spearman. La dimensión instrumentalidad y el desarrollo de capacidades conceptuales, procedimentales y actitudinales por medio de la prueba de Spearman tuvieron correlación directa moderada en todas ellas ( $\mathrm{p}<0,001)$ con valores del índice rho de Spearman de 0,524, 0,481 y 0,503 respectivamente. Conclusiones: La percepción de los estudiantes sobre la estrategia de aprendizaje basado en problemas se correlaciona positivamente con el desarrollo de capacidades.

Palabras clave: Aprendizaje basado en problemas; Educación basada en competencias; Percepción.

\begin{abstract}
Objective: To determine the relationship between the students' perception of the use of problem-based learning based and the development of abilities in the Faculty of Stomatology of the Inca Garcilaso de la Vega University (UIGV). Materials and method: Descriptive, cross-sectional and correlational study. The population was students of the fifth cycle the Faculty of Stomatology of the UIGV of 2015-I, that were taking removable partial prosthesis and who met the inclusion criteria (enrolled in the subject of Removable Partial Prosthesis of the year 2015-I, accessibility, availability and acceptance of participation in the study). We worked with 49 students. The instrument used: An evaluation questionnaire. The tenor of the questions inquires about the perception that the student develops on the methodology problem-based learning and the development of abilities that considers to have developed. Results: No correlation was found between
\end{abstract}

\section{Artículo Original}

Víctor Fernando Lamas Lara ${ }^{1, a}$, Dora Noelia Gómez Meza 2,a

${ }^{1}$ Facultad de Ciencias de la Salud. Universidad Peruana Los Andes. Lima, Perú.

2 Facultad de Odontología. Universidad Nacional Mayor de San Marcos. Lima, Perú.

a Magíster en Estomatología.

\section{Correspondencia:}

Víctor Fernando Lamas Lara

Correo electrónico: VFLLMAT@gmail.com Jr. Santa Honorata 415 Pando Tercera Etapa. Lima 1, Perú.

\section{Coautor:}

Dora Noelia Gómez Meza

dgomezm@unmsm.edu.pe

Conflicto de intereses: Los autores declaran no tener conflictos de interés.

Fuente de financiamiento: Autofinanciado.

Fecha de recepción: 03/02/17

Fecha de aceptación: 25/08/17

(C) Los autores. Este artículo es publicado por la revista Odontología Sanmarquina de la Facultad de Odontología, Universidad Nacional Mayor de San Marcos. Este es un artículo de acceso abierto, distribuido bajo los términos de la licencia Creative Commons Atribucion - No Comercia_Compartir Igual 4.0 Internacional. (http://creativecommons.org/licenses/by-nc-sa/4.0/) que permite el uso no comercial, distribución y reproducción en cualquier medio, siempre que la obra original sea debidamente citada. 
the dimension conditions on the use of the didactic model of learning with the development of conceptual, procedural and attitudinal abilities by means of the Spearman correlation test. The instrumental dimension and the development of conceptual, procedural and attitudinal abilities by the Spearman test had a moderate direct correlation in all of them ( $\mathrm{p}<0.001$ ) with Spearman rho index values of $0.524,0.481$ and 0.503 respectively. Conclusions: The students' perception of problem based learning strategy correlates positively with the development of abilities.

Keywords: Competency-based education; Perception; Problem-based learning.

\section{Introducción}

La universidad ha tenido que afrontar dos grandes retos: identificar y definir los resultados de aprendizaje relevantes en un mundo en constante transformación; y el cómo promover una participación del estudiante en la construcción de su proceso de aprendizaje a lo largo de la vida y en la aplicación del conocimiento en los diferentes entornos del mundo real La educación superior debe dirigirse a la formación de profesionales competentes: con habilidades para resolver problemas, analizar, enjuiciar y tomar decisiones y participar en la investigación respecto de su propia práctica.

Ante tales retos, es urgente un enfoque curricular integral en el que el aprendizaje autónomo y la capacidad de los estudiantes para resolver problemas se sitúen al centro de la acción educativa ${ }^{1,2}$.

El aprendizaje basado en problemas (ABP) como estrategia de aprendizaje ha tenido una rápida extensión por los Estados Unidos y poco después por Europa postulándose como una de las innovaciones más importantes en el entorno académico de las ciencias de la salud.

El estudio de las competencias en el proceso de formación de los futuros profesionales, es un instrumento de valor creciente, ya sea para investigar su adquisición, desarrollo y fortalecimiento, como también la repercusión de éstas en el desempeño laboral ${ }^{3,4}$.

El ABP, en inglés llamado PBL (Problem-Based Learning), es una estrategia de enseñanza basada en el estudiante como protagonista de su propio aprendizaje ${ }^{5}$. Así, el valor del aprendizaje de conocimientos está en el mismo nivel que la adquisición de habilidades y actitudes. Así los estudiantes consiguen elaborar un diagnóstico de las necesidades de aprendizaje, construir el conocimiento de la materia y trabajar cooperativamente ${ }^{6,7}$.

Los resultados obtenidos durante el proceso de enseñanza-aprendizaje se relacionan directamente con el grado de eficiencia de las estrategias planificadas. El diseño de tales estrategias debe facilitar el aprendizaje significativo y la evaluación continua del hecho educativo será el aspecto determinante de la excelencia educativa ${ }^{8}$. En verdad, se viene constatando, los beneficios educativos del $\mathrm{ABP}$, la congruencia entre $\mathrm{ABP}$ y la carpeta de aprendizaje, y las posibilidades de extender el modelo aplicado al conjunto de la titulación, y responde a las exigencias actuales de la educación superior, el conocimiento está cambiando muy rápidamente; y se considera, la necesidad de: hacer menos énfasis en acu- mular muchos datos y dar más importancia en adquirir destrezas, competencias y habilidades. Es decir, facilitar el desarrollo de capacidades generales, cualidades personales y profesionales ${ }^{9,10}$.

El estudio tuvo por objetivo determinar la relación existente entre la percepción de los estudiantes sobre el uso del modelo didáctico aprendizaje basado en problemas y el desarrollo de capacidades en la formación del odontólogo.

\section{Materiales y método}

Se realizó un estudio de tipo descriptivo y transversal. La población de estudio estuvo constituida por los estudiantes del quinto ciclo de la Facultad de Estomatología de la Universidad Inca Garcilaso de la Vega del 2015I, de la Asignatura de Prótesis Parcial Removible y que cumplieron con los criterios de inclusión (matriculados en la asignatura de Prótesis Parcial Removible del año 2015-I, accesibilidad, disponibilidad horaria y aceptar participar en el trabajo). En cuanto al interés del investigador de analizar una muestra concreta (verificar si la innovación didáctica funciona en una clase) el tamaño de la muestra se consideró el tamaño del grupo objeto de estudio (49 estudiantes). El ABP es una herramienta para el aprendizaje significativo, implicó tareas que sean un reto intelectual, basados en la investigación, el debate y las presentaciones orales. Por ello, al comenzar a desarrollar cada unidad de trabajo se les planteaba problemas específicos de mediana a gran complejidad relacionados con el contenido temático de cada unidad que se podían presentar no sólo en su desempeño pre-clínico sino clínico de tal manera que el estudiante debía realizar no sólo la búsqueda bibliográfica, sino consultar con sus pares, con el docente responsable del estudio e inclusive con otros docentes del área sobre la posible solución o soluciones al problema planteado. La actividad propia del docente responsable del estudio se orientó a crear un ambiente de aprendizaje modificando los espacios, dando acceso a la información, modelando y guiando el proceso, en las sesiones cumplidas durante el semestre académico: activación, investigación, resolución y evaluación.

Se utilizó como técnica de recolección de datos la encuesta, y como instrumento un cuestionario de 23 preguntas para evaluar la percepción de los estudiantes en relación al uso del ABP y el desarrollo de capacidades de la siguiente forma: 1. Percepción del ABP (a la que se le asignaron ocho preguntas), en relación a condi- 
ciones (cuatro preguntas con referencia a motivación, implicación, gestión del tiempo, autopercepción de desarrollo de capacidades) e instrumentalidad (cuatro preguntas en referencia a practicidad, ventajas, aplicabilidad y utilidad para la futura práctica profesional); 2. Desarrollo de capacidades; a las que se le asignaron 15 preguntas: conceptuales (seis preguntas referidas a organización, gestión de información, toma de decisiones, razonamiento crítico, resolución de problemas, aprendizaje autónomo); procedimentales (cuatro preguntas en relación a trabajo en equipo, aplicación de conocimiento para diagnóstico y tratamiento, aplicación de instrumental) y actitudinales (cinco preguntas que veían las relaciones interpersonales, iniciativa, responsabilidad, compromiso ético, adaptación a nuevas situaciones). Cada pregunta tenía un puntaje mínimo de uno si consideraba que no era relevante y un puntaje máximo de cuatro si consideraba que era de mucha importancia la percepción del ABP en el desarrollo de capacidades, lo cual permitió por cada ítem, por cada dimensión y a la vez por cada variable obtener valores mínimos, máximos y medianos (Q2). El cuestionario se validó por medio del juicio de expertos y una prueba piloto en la cual se evaluó la consistencia interna por medio del alfa de Cronbach $(0,79$ y 0,93$)$ para las variables de estudio: percepción de los estudiantes y desarrollo de capacidades.

El procesamiento y análisis estadístico de la información se realizó a través del programa estadístico SPSS versión 21. Para relacionar las variables de estudio y sus dimensiones se aplicó la prueba de correlación de Spearman, calculando el coeficiente de correlación respectivo. Las pruebas fueron trabajadas a un nivel de significancia de $5 \%{ }^{11}$.

\section{Resultados}

Respecto a la percepción de los estudiantes sobre el uso del ABP (Tabla 1) se encontró que la dimensión condiciones obtuvo un puntaje de entre 7 y 13, siendo el valor mediano (Q2) 9, mientras que la mayoría de los indicadores tuvo un valor mediano de 2 ; en cuanto a la dimensión desarrollo de instrumentalidad, el puntaje obtenido estuvo entre 4 y 12, siendo el valor mediano 7 y todos sus indicadores tuvieron un valor mediano de 2. La percepción total de esta estrategia de enseńanza y aprendizaje estuvo entre un puntaje de 11 y 23 con un valor mediano de 16 .

Respecto al desarrollo de capacidades por el uso del ABP (Tabla 2) se encontró un nivel de desarrollo semejante en las tres dimensiones conceptual, procedimental y actitudinal obteniéndose valores medianos de 10, 8 y 9 respectivamente. En cada uno de sus indicadores el valor mediano fue 2 y en casi todos los indicadores los valores mínimo y máximo observados fueron 1 y 3 . La percepción del desarrollo de capacidades total estuvo entre un puntaje de 17 y 45 con un valor mediano de 26.

Cuando se relacionó las condiciones sobre el uso del ABP con el desarrollo de capacidades conceptuales, procedimentales y actitudinales por medio de la prueba de correlación de Spearman no se encontró correlación en ninguna de ellas $(p>0,05)$.

Cuando se relacionó el desarrollo de instrumentalidad sobre el uso del modelo didáctico de ABP con el desarrollo de capacidades conceptuales, procedimentales y actitudinales por medio de la prueba de correlación de Spearman se encontró correlación directa moderada en todas ellas $(\mathrm{p}<0,001)$ con valores del índice rho de Spearman de $0,524,0,481$ y 0,503 respectivamente.

Tabla 1. Percepción sobre el uso del modelo didáctico de aprendizaje basado en problemas por cada uno de los indicadores y el total

\begin{tabular}{|c|c|c|c|c|c|c|c|c|}
\hline ABP* & Indicador & Ítem & $\mathrm{n}$ & Mínimo & Q1 & Q2 & Q3 & Máximo \\
\hline \multirow[t]{7}{*}{ Condiciones } & $\begin{array}{l}\text { Apreciación sobre el desarrollo de capacidades } \\
\text { en el alumno }\end{array}$ & 1 & 49 & 2 & 2 & 3 & 4 & 4 \\
\hline & $\begin{array}{l}\text { Desarrollo de motivación para realizar la } \\
\text { actividad }\end{array}$ & 2 & 49 & 1 & 1 & 2 & 2 & 3 \\
\hline & $\begin{array}{l}\text { Implicancia del alumnado en actividades del } \\
\text { ABP }\end{array}$ & 3 & 49 & 1 & 2 & 2 & 3 & 4 \\
\hline & $\begin{array}{l}\text { Tiempo adecuado para la realización de las } \\
\text { actividades }\end{array}$ & 4 & 49 & 1 & 2 & 2 & 3 & 4 \\
\hline & Dimensión condiciones & $1-4$ & 49 & 7 & 8 & 9 & 10 & 13 \\
\hline & Presenta practicidad para el trabajo del curso & 5 & 49 & 1 & 1 & 2 & 2 & 3 \\
\hline & Presenta ventaja sobre el método tradicional & 6 & 49 & 1 & 1,5 & 2 & 2 & 3 \\
\hline \multirow{3}{*}{$\begin{array}{l}\text { Desarrollo de } \\
\text { Instrumentalidad }\end{array}$} & Aplicabilidad para otros cursos de especialidad & 7 & 49 & 1 & 1 & 2 & 2 & 4 \\
\hline & $\begin{array}{l}\text { Utilidad para su futuro profesional de las } \\
\text { actividades del ABP }\end{array}$ & 8 & 49 & 1 & 1 & 2 & 2 & 3 \\
\hline & Dimensión desarrollo de instrumentalidad & $5-8$ & 49 & 4 & 6 & 7 & 8,5 & 12 \\
\hline Total & & $1-8$ & 49 & 11 & 15 & 16 & 18 & 23 \\
\hline
\end{tabular}

* $\mathrm{ABP}=$ Aprendizaje basado en problemas 
Cuando se relacionó las dos variables de estudio en su conjunto, por medio de la prueba de correlación de Spearrman, también se encontró correlación directa moderada $(\mathrm{p}=0,002)$ con valores del índice rho de Spearrman de 0,433.

\section{Discusión}

Prieto et al. ${ }^{12}$ asumiendo el enfoque de aprendizaje activo señalan que "el Aprendizaje Basado en Problemas representa una estrategia eficaz y flexible que, a partir de lo que hacen los estudiantes, puede mejorar la calidad de su aprendizaje universitario en aspectos muy diversos". Así, el ABP ayuda al estudiante a desarrollar y a trabajar diversas competencias; el presente estudio reflejó que el $\mathrm{ABP}$ se relaciona positiva y significativamente con el desarrollo de capacidades, y que niveles moderados-bajos de ABP le corresponde niveles moderados-bajos de capacidades ${ }^{13}$.

Galindo et al. ${ }^{14}$ reportan que se permitió el desarrollo de competencias analíticas, argumentativas, propositivas, sociales e interpersonales con base en la búsqueda planificada de la información para la solución de problemas, sin embargo, en este estudio se encontró que el promedio del desarrollo de capacidades fue 37,5 en un rango de 15 a 60 , que lo ubica casi 10 puntos por debajo de una media teórica, indicando niveles bajos de la mencionada variable, relacionada básicamente con la interiorización de conocimientos (según esto, el ABP no es transmisión mecánica del conocimiento), por ello, no ha resultado ser tan fácil de transmitir, quizá se deba a que no es algo que puede transmitirse, debido a que el profesor no lo tiene elaborado para ser utilizado por los estudiantes, sino que los estudiantes lo construyen en su proceso de aprendizaje.

La instrumentalidad del ABP, como se comprobó en este trabajo, está relacionado con el desarrollo de capacidades actitudinales, lo cual permite afirmar que el ABP estimula el sentido de la participación colaborativa a través de los equipos de trabajo, fortaleciendo y ampliando las habilidades de los estudiantes para entablar relaciones interpersonales ${ }^{15}$. Como se mencionó en la metodología de este estudio, al haberse planteado problemas de mediana a gran complejidad se hizo necesario que el estudiante interactúe y participe con sus compañeros en la solución y el desarrollo de los mismos.

El componente instrumentalidad del ABP es el que aporta casi la totalidad de la relación explicando la varianza en un $32,2 \%$ de la relación ABP-Instrumentalidad con el desarrollo de capacidades, cuando es considerado solo. Lo cual concuerda con lo encontrado por Amato y Novales ${ }^{16}$, quienes realizaron un estudio con el objetivo de conocer el grado en que los estudiantes

Tabla 2. Desarrollo de capacidades

\begin{tabular}{|c|c|c|c|c|c|c|c|c|}
\hline $\begin{array}{l}\text { Desarrollo de } \\
\text { capacidades }\end{array}$ & Indicador & Ítem & $\mathbf{n}$ & Mínimo & Q1 & Q2 & Q3 & Máximo \\
\hline \multirow{7}{*}{ Conceptuales } & Capacidad de organización y clasificación & 9 & 49 & 1 & 1 & 2 & 2 & 3 \\
\hline & Capacidad de gestión de la información & 10 & 49 & 1 & 1 & 2 & 2 & 3 \\
\hline & Resolución de problemas & 11 & 49 & 1 & 1 & 2 & 2,5 & 3 \\
\hline & Toma de decisiones & 12 & 49 & 1 & 1 & 2 & 2 & 3 \\
\hline & Razonamiento crítico & 13 & 49 & 1 & 1 & 2 & 2 & 3 \\
\hline & Aprendizaje autónomo & 14 & 49 & 1 & 1 & 2 & 2 & 3 \\
\hline & Dimensión conceptual & $9-14$ & 49 & 6 & 9 & 10 & 13 & 18 \\
\hline \multirow{5}{*}{ Procedimentales } & Trabajo en equipo & 15 & 49 & 1 & 1 & 2 & 3 & 3 \\
\hline & Aplicación de conocimientos para el diagnostico & 16 & 49 & 1 & 1 & 2 & 2 & 3 \\
\hline & Aplicación de materiales e instrumentales & 17 & 49 & 1 & 1 & 2 & 2 & 3 \\
\hline & Aplicación adecuada para el tratamiento & 18 & 49 & 1 & 1 & 2 & 2 & 3 \\
\hline & Dimensión procedimental & $15-18$ & 49 & 4 & 6 & 8 & 9 & 12 \\
\hline \multirow{6}{*}{ Actitudinales } & Habilidades en las relaciones interpersonales & 19 & 49 & 1 & 1 & 2 & 2 & 3 \\
\hline & Adaptación a nuevas situaciones & 20 & 49 & 1 & 1 & 2 & 2 & 3 \\
\hline & Iniciativa y creatividad & 21 & 49 & 1 & 1 & 2 & 2 & 4 \\
\hline & Responsabilidad & 22 & 49 & 1 & 1 & 2 & 2 & 3 \\
\hline & Compromiso ético & 23 & 49 & 1 & 1 & 2 & 3 & 3 \\
\hline & Dimensión actitudinal & $19-23$ & 49 & 5 & 7,5 & 9 & 11 & 15 \\
\hline Total & & $9-23$ & 49 & 17 & 23 & 26 & 33,5 & 45 \\
\hline
\end{tabular}


aceptaron y consideraron útiles las técnicas del ABP y su evaluación entre pares. Encontraron que la mayoría de los estudiantes consideró que el ABP fue útil (82\%) y que las actividades de evaluación entre pares fueron justas y formativas (70\%).

Por otro lado, la interacción de los dos componentes del ABP explica sólo un 30,7\% de la relación. Lo que indica que la dimensión de las condiciones para el ABP no se relaciona con el desarrollo de capacidades, lo cual concuerda con los resultados de este estudio, en la cual, de las dos dimensiones evaluadas del ABP, sólo la instrumentalidad presentó correlación positiva con las tres dimensiones del desarrollo de capacidades.

Por lo que se sostiene que habría que ir más allá del debate de si el ABP es o no efectivo, asumiendo la variabilidad inherente a esta técnica, y enfocar las futuras investigaciones hacia los procesos que subyacen a la estrategia del ABP, es decir, una investigación guiada por la teoría cognitiva y educativa, y no centrada en cuestiones relacionadas con su implementación ni con su efectividad, e investigar con muestras amplias y con el ABP integrado en todo el currículo. Además de diversificar los instrumentos de medición y evaluación.

El ABP supone un proceso relativamente complejo que como todas las metodologías tiene sus dificultades, especialmente al iniciarse en su aplicación. Pero también, mejora la satisfacción con el aprendizaje y prepara mejor a los estudiantes para afrontar situaciones reales que se encontrarán en su futuro laboral ${ }^{17}$.

\section{Conclusiones}

- La percepción de los estudiantes sobre el uso del modelo didáctico de ABP se correlaciona positivamente con el desarrollo de capacidades en la muestra estudiada.

- El desarrollo de la instrumentalidad sobre el uso de la estrtategia del ABP se correlaciona positivamente con el desarrollo de capacidades conceptuales, procedimentales y actitudinales en la muestra estudiada.

- Las condiciones sobre el uso de la estrategia del ABP no están correlacionadas con el desarrollo de capacidades conceptuales, procedimentales ni actitudinales en la muestra estudiada.

\section{Referencias bibliográficas}

1. Reverte J, Gallego A, Molina, R, Satorre R. El aprendizaje basado en proyectos como modelo docente: experiencia interdisciplinar y herramientas groupware. Proyecto de innovación tecnológico-educativo e innovación educativa de la Universidad de Alicante; XIII Jornadas de Enseńanza Universitaria de la Informática, JENUI'07, Teruel: libro de actas. Madrid: Thomson Paraninfo, 2007; 285-292.

2. Mioduser D, Betzer N. The contribution of project-based learning to high achievers' acquisition of technological knowledge. Int J Technol Des Educ. 2008;18:59-77. doi:10.1007/s10798-006-9010-4.
3. Saavedra E, Illescas M, Cabezas M. Aprendizaje basado en problemas (ABP) como estrategia para adquisición de competencias genéricas: estudiantes de nutrición y dietética, Universidad de La Frontera. Rev Chil Nutr. 2014;41(2):167-172.doi: 10.4067/S071775182014000200007.

4. Molina I, Fernández A, Pastoriza P, Bravo I, Herrero R. Aplicación del aprendizaje basado en problemas (ABP) a la operación básica de evaporación. Edusfarm. 2008;3:27.

5. Hernández L, Hernández E. Mejoras en el logro de competencias en el aprendizaje de anatomía, mediante la aplicación del método Aprendizaje Basado en Problemas (ABP). En Congreso Iberoamericano de Ciencia, Tecnología, Innovación y Educación. Buenos Aires, Argentina; 2014.

6. Arends I. Learning to Teach. $9^{\text {th }}$.Ed. New York: McGraw Hill; 2009.

7. De Juanas A, Fernández M. Competencias y estrategias de aprendizaje. Reflexiones sobre el proceso de cambio en el EESS. Cuadernos de Trabajo Social. 2008;21:217230.

8. Morales P. Estadística aplicada a las Ciencias Sociales. Madrid. Universidad Pontificia Comillas. 2012.

9. Albanese M, Mitchell S. Problem-based learning: a review of literature on its outcomes and implication issues. Acad Med. 1993;68(1):52-81.

10. Prieto A, Barbarroja J, Reyes E, Monserrat J, Díaz D, Villarroel M, Álvarez M. Un nuevo modelo de aprendizaje basado en problemas, el ABP 4x4, es eficaz para desarrollar competencias profesionales valiosas en asignaturas con más de 100 alumnos. Aula Abierta. 2006;87,171194.

11. Prieto A. (2007). Enseńanza y aprendizaje en la Universidad del futuro. 2007. Disponible en http://www.problembasedlearning.eu/

12. Gómez F, Rivas I, Mercado F, Barjola P. Aplicación interdisciplinar del Aprendizaje Basado en Problemas (ABP) en Ciencias de la Salud: Una herramienta útil para el desarrollo de competencias profesionales. Red U. 2009;4:1-19.

13. Koh G, Khoo H, Wong M, Koh D. The effects of problem-based learning during medical school on physician competency: a systematic review. CMAJ. 2008; Jan 1; 178 (1):34-41. doi: 10.1503/cmaj.070565.

14. Morales P, Landa V. Problem based Learning. Theoria. 2004;13:145-157.

15. Galindo I, Arango M, Díaz D, Villegas E, Aguirre C, Kambourova M, Jaramillo P. ¿Cómo el aprendizaje basado en problemas (ABP) transforma los sentidos educativos del programa de Medicina de la Universidad de Antioquia? Iatreia. 2011;24(3):325-334.

16. Rodriguez J. Aprendizaje basado en problemas. Buenos Aires: Médica Panamericana; 2004.

17. Amato D, Novales X. Aceptación del Aprendizaje Basado en Problemas y de la evaluación entre pares por los estudiantes de medicina. Gac Med Mex. 2009;145(3):197205. 